\title{
Impact Assessment of the Power Line Channel Characteristics in Real Topology
}

\author{
Dženana TOMAŠEVIĆ ${ }^{*}$ Tatjana KONJIĆ, Suad KASAPOVIĆ
}

\begin{abstract}
This paper investigates the effect of different parameters of power line topology on PLC system. Information about real radial power distribution network is used to define a PLC channel model in NS-3 simulator. The overhead LV distribution network was modelled in the extended bandwidth. Results of real measurements are linked with simulation results in order to see which parameters influence the communication significantly.
\end{abstract}

Keywords: Narrowband power line communications; overhead lines; power line channel; real radial power distribution network

\section{INTRODUCTION}

Smart grid and its widely used applications (automatic meter reading, load control, remote diagnostics, etc.) require reliable communication infrastructure. PLC is a technology used primarily for remote metering by utility companies. It has recently experienced increased deployment because it is cost effective since it reuses power lines for the purpose of data communication. Despite all advantages, power lines were not traditionally designed for data transmission so PLC has to deal with phenomena such as the presence of noise and signal attenuation [1]. Also, continuous connection and disconnection of different loads (variations of topology), EMI, NIE generated by regular appliances (such as TVs, boilers, etc.) and novel equipment (such as DG devices, EVs or battery charges) highly affect the communication performance [2]. As a result, many researches and utility companies are motivated for continuous technology improvement and validation of this technology. PLC has been active research area in the last couple of years and is still attractive and live research topic [3].

PLC systems fall into three areas [4]:

- UNB operates at a very low data rate $(100 \mathrm{bps})$ in the low frequency band $(0.3-3 \mathrm{kHz})$. UNB uses one-way communication, used in particular for load control. UNB has a very large operational range (hundreds of kilometres).

- $\quad \mathrm{NB}$ operates in the frequency band 3 - $500 \mathrm{kHz}$ (3 $148.5 \mathrm{kHz}$ in Europe). NB PLC systems can be further divided into LDR NB PLC, employing single carrier modulations for throughputs of hundreds of bps to few kbps and HDR NB PLC, employing multiple carrier modulations for throughputs up to hundreds of kbps.

- $\quad$ BB operates in the high frequency band (1.8 - 30 (250) $\mathrm{MHz}$ ) and has data rates of several Mbps up to hundreds of Mbps.

There are two areas of application of these systems: indoor and outdoor. Indoor deployment refers to systems inside the building (behind the power meter) and outdoor systems connect, through a transformer, a residential area in which PLC networks are installed [5]. Both systems have their own communication requirements.

According to previous studies, NB PLC has been used in smart metering applications in Europe [6]. Many communication protocols have been developed according to standards that include the European Committee for Electrotechnical Standardization CENELEC band (from 9 to 148.5
$\mathrm{kHz}$ ), the US FCC band (from 10 to $490 \mathrm{kHz}$ ) and the Japanese ARIB band (from 10 to $450 \mathrm{kHz}$ ) [7]. In Europe, CENELEC A band is reserved for electricity suppliers and distributors and is compliant with EN 50065 as harmonized standard [8]. New services and applications, along with potential problems listed before, are reasons for starting to consider band extension in Europe. There are many research papers covering performance aspects of NB PLC systems in extended bandwidth. Authors in [9] describe channel measurement methods in the frequency band $50-500 \mathrm{kHz}$, including channel, noise and impedance measurements in Korean residential and industrial electricity grids. These measurements were performed for underground LV power lines. The increase of bandwidth and the robustness against disturbances were discussed in [10] where the performance of NB PLC technology was checked. Authors in [11] analysed the noise characteristics in time-frequency representation. They also measured the transfer function in five different sites in several LV networks in France. The channel capacity was estimated in order to support new IoT applications and services. Results for performance analysis of PRIME-based NB PLC system for the rural distribution grid were presented in [12]. The error performance is correlated with the measured PLC channel frequency response and noise characteristics. The measurements in real topology were conducted in [13] where the possibility of BB PLC and NB PLC communication with/without repeaters was investigated. Authors in [14] compared the performances of mono carrier and multicarrier modulations in terms of BER. They focused on the FSK, SFSK and OFDM systems widely used in NB PLC technology. The transfer function of LV NB PLC channel using Zimmermann's model was compared with the transfer function obtained from TLT based model in [15]. Verification of both models was done over the frequency range 3 - $500 \mathrm{kHz}$.

Nowadays, research efforts are also focused on developing different PLC software modules suitable for the simulation of PLC networks. The key parameters for evaluation of the communication performance are data rate, robustness, noise immunity, achievable distances and adaptation to network changes [13]. In order to reach these parameters, it is necessary to carry out certain measurements which are often quite expensive. On the other hand, the simulator enables free configuration of the PLC network topology and is flexible enough to capture the time and frequency selective behaviour of PLC channels 
[16]. Several simulators (Canete, FTW, etc.) were designed in MATLAB $[17,18]$. Other researchers use the advanced simulation tools, such as OMNET++, OPNET and NS-3 [19]. According to [20], several software modules have been developed in NS-3. These are modules for the generation of channel transfer functions and noise in PLC network based on TLT. The simulator accepts user-defined topologies and has libraries for a large number of communication technologies. It is suitable for implementation of both NB and $\mathrm{BB}$ communication.

According to published articles review in this area, it could be noticed that there is a lack of research that links real field measurements with simulation results, simulated networks are usually the radial networks with small number of nodes and the observed low voltage networks contain underground cables.

This article focuses on the radial LV network which consists of overhead cables. Information about real network topology (number and length of branches, type and cross section of cables, etc.) are used to define a PLC channel model in NS-3 simulator. The overhead LV distribution network was modelled in the frequency band $0-500 \mathrm{kHz}$. The effect of different parameters of power line topology on PLC system such as terminal load impedances, direct line length from transmitter to receiver, cable type, background noise and modulation type was investigated.

The main contributions of this paper are the following: 1. Results from simulation and available real-time measurements are linked to show that there are different interference factors which affect the reliability of communication.

2. The observed and modelled network consists only of overhead lines.

3. The effect of different parameters of power line topology on PLC system in the frequency band $0-500 \mathrm{kHz}$ was investigated in the real radial LV distribution network with 69 nodes.

The paper is organized as follows. In the second section powerline channel modelling is briefly described. Simulation results of the real radial power distribution network with special attention paid on influence of the terminal load impedance, influence of the cross section and line type, influence of the distance between the transmitter and the receiver and influence of background noise and modulation type are presented in the third section. Measurements in real power network are given in the fourth section. The discussion of obtained results is presented in the fifth section and finally conclusions are in the sixth section.

\section{POWERLINE CHANNEL MODELLING}

As mentioned above, the power line channel is a very harsh and noisy transmission medium that is very difficult to model [21]. For the purpose of establishing a reliable communication system, it is very important to determine the characteristics of the channel. There are two basic approaches for modelling the distribution line communication channel. The first approach, also known as top-down, describes the multipath propagation via an echo model in the time or frequency domain [22]. This modelling approach is often used when the topology is unknown. It is quite easy to implement this solution but dependence on the measurement accuracy is often a big drawback. The second approach, also known as bottom-up, requires the knowledge about topology and cable types. It describes the relationship between the network behaviour and the model parameters. The CTF can be obtained by both approaches (top-down and bottom-up) and for different types of the network topologies. There are two main topology structures, the radial and the mesh grid topology. In the radial grid topology there are no closed loops and, therefore, radial power network is vulnerable to a power line disconnection. This problem does not occur with the mesh grid topology because there are alternative connections between nodes, so communication is possible via multiple alternative routes. The proposed method in this article, based on scattering matrices, is not simply applicable to mesh grid topology because this topology cannot be divided into subnetworks, which significantly increases the computational complexity and errors in the frequency domain. According to [23], there is a novel channel modelling method that is applicable to various topologies. It involves placing all necessary information nodes into the network and converting the problem of determining the transmission characteristic between signal source and each receiver into a problem of determining the transmission characteristic between information node and the information node related to each receiver. However, the radial grid topology is mostly used in LV networks, while the mesh grid topology is usually used in MV and HV networks. In this paper, the bottom-up approach based on TLT principles is used. Power lines and all passive elements are presented as two-port networks described by their ABCD parameters [24]. In this case, the channel is modelled as two conductor transmission line. The PLC simulator in NS-3 accepts user-defined grid topologies and is used to generate channel transfer functions and noise spectral densities. From these results, SNR and channel link capacity are derived.

Channel transfer function. The channel transfer function of the power line channel can be calculated after the topology is created. After the shortest path between the transmitter and the receiver is determined, the topology is divided into smaller subunits (two-port networks). Then the input impedance and the CTF are calculated for each subunit. The overall CTF can be computed using the impedance carry-back method and the overall ABCD matrix using the equation:

$$
\begin{aligned}
& H(f)=\frac{V_{2}(f)}{V_{1}(f)}= \\
& =\frac{Z_{S}+Z_{L}}{A(f) \cdot Z_{L}+B(f)+C(f) \cdot Z_{S} \cdot Z_{L}+D(f) \cdot Z_{S}}
\end{aligned}
$$

where $A, B, C$ and $D$ are frequency-dependent coefficients, which are calculated from the secondary parameters, $Z_{s}$ is source impedance of the transmitter and $Z_{L}$ is load impedance of the receiver (see Fig. 1) [25].

In NS-3 PLC simulator, CTF is calculated via a deterministic frequency domain method and presents a ratio of the load voltage to the source voltage. The analysis of the CTF is needed in order to evaluate the performance of the PLC system. 


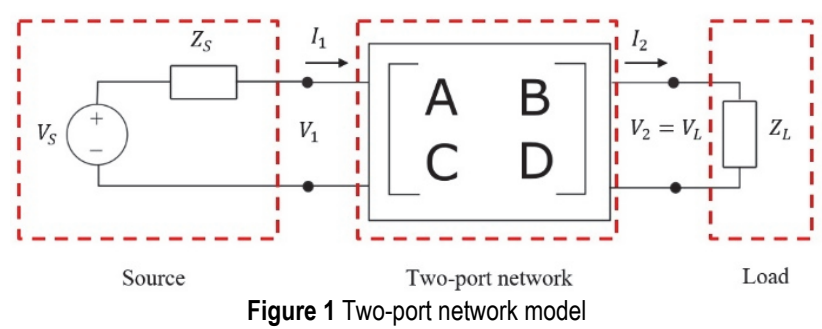

Channel link capacity. The Shannon capacity is the common metric used to determine the theoretical achievable rate limit [26]. Shannon's Theory describes the maximum transmission rate at which can be transmitted information over a specific bandwidth of communication channel and is given by:

$$
C=B \cdot \log _{2}\left(1+\frac{S}{N}\right)
$$

where $C$ is the maximum channel capacity, $B$ is the channel bandwidth, and $S / N$ is the received signal power to the noise signal power at the receiver.

If the channel is divided into $N$ subchannels (subbands), then the channel capacity for each subband can be calculated using the equations:

$$
\begin{aligned}
& C=\int_{B} \log _{2} \cdot\left(1+\frac{\Phi_{R R}(f)}{\Phi_{N N}(f)}\right) \\
& C=\int_{B} \log _{2} \cdot\left(1+\frac{\Phi_{T T}(f) \cdot|H(f)|^{2}}{\Phi_{N N}(f)}\right)
\end{aligned}
$$

According to Eq. (3) and Eq. (4), received signal varies with the CTF. In order to compute the channel capacity, it is necessary to know the PSD on the receiver side $\left(\Delta \Phi_{R R}(f)\right)$, the transmitter side $\left(\Delta \Phi_{T T}(f)\right)$ and the interference $\left(\Delta \Phi_{N N}(f)\right)$. The total channel capacity can be calculated by adding the capacities of the individual subbands and multiplying by the subband width $\Delta f=B / N$ :

$$
C=\Delta f \cdot \sum_{v=1}^{N} \log _{2} \cdot\left(1+\frac{\Phi_{T T}(v) \cdot|H(v)|^{2}}{\Phi_{N N}(v)}\right)
$$

In order to compute the maximum channel capacity in NS-3 PLC simulator, it is necessary to know the type of modulation and a number of modulation states.

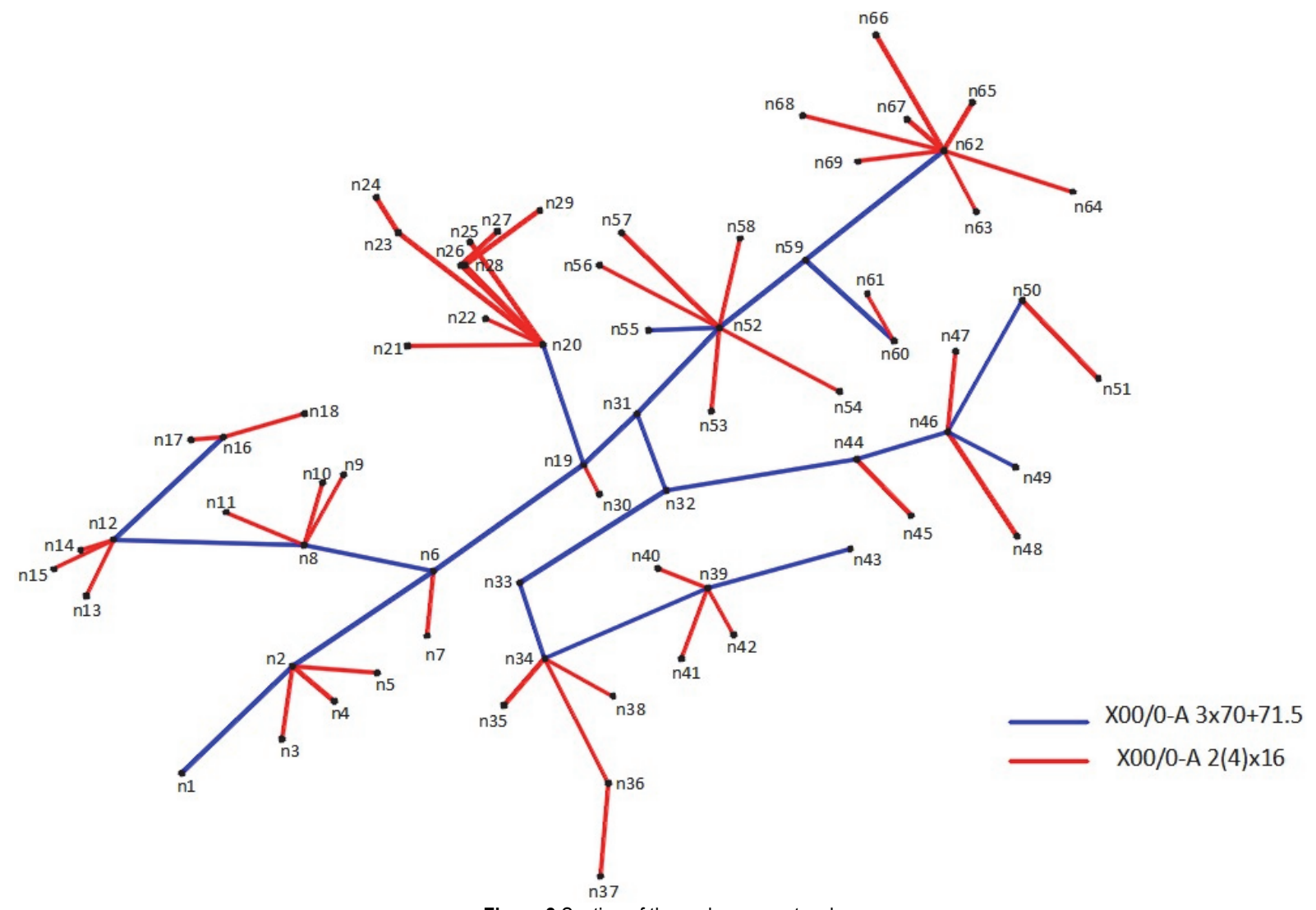

Figure 2 Section of the real power network

\section{SIMULATION RESULTS-REAL POWER NETWORK}

As it was mentioned earlier, LV power lines can be overhead or underground cables. There are many research papers dealing with the influence of different power line topology parameters on the CTF and channel link capacity
$[3,11,16,25]$ but only a few consider overhead lines and discuss performance assessment on PLC system [8, 13]. Fig. 2 shows the section of the real radial power distribution network used for simulations. The node $\mathrm{n} 1$ presents TS with a data concentrator in it and also a transmitting node in simulations. The other nodes are 
power meters at the entrance of the houses or fuse boxes. The network is in a suburb area of the city where overhead LV network is dominant. The overhead cables are aluminium twisted distribution wire with cross section 16 $\mathrm{mm}^{2}$ (red line) and $70 \mathrm{~mm}^{2}$ (blue line). Each wire has the insulation which is made of cross-linked polyethylene XLPE. These cables are often used in distribution network.

In order to investigate the influence of different topology parameters on the CTF and channel link capacity, several simulation scenarios were considered in the NS-3 PLC simulator. Simulations were performed for narrowband technology over the extended frequency range 0 - $500 \mathrm{kHz}$. The 64PSK modulation was used. Transmitter impedance was $40 \Omega$, receiver impedance $100 \Omega$ and node impedance was $300 \Omega$. Transmitting power was $1 \mathrm{e}-8$ $\mathrm{mW} / \mathrm{Hz}$. Background noise was set according to [27] on the value of $1 \mathrm{e}-9 \mathrm{~mW} / \mathrm{Hz}$. All values of the transmission capacities of the line are determined on the basis of theoretical calculations of the simulator. This is the transmission capacity on the physical layer.

\subsection{Influence of the Terminal Load Impedance}

For LV distribution networks loads are presented by houses which are a superposition of several electrical loads [28]. According to [29], the loads can be classified into three groups: approximately constant impedances, timeinvariant but frequency-selective impedances and timevarying and frequency-selective impedances. In the real power network the value of the impedance depends on the connected electrical devices as well as on the frequency. However, to illustrate better the effects of the physical channel in the network performance, all the loads have been considered static and equal [30]. Two scenarios were considered. In the first scenario, the node n68 was a receiver node and in the second scenario, the n45 was a receiver node. These nodes were chosen because they are far from the transmitting node. The distance between the transmitting and the receiving node is $278 \mathrm{~m}$ in the first scenario and $229 \mathrm{~m}$ in the second scenario. In both cases, the load impedance was varying from low to open-circuit impedance $\left(5,50,1000,10^{8}\right)$.

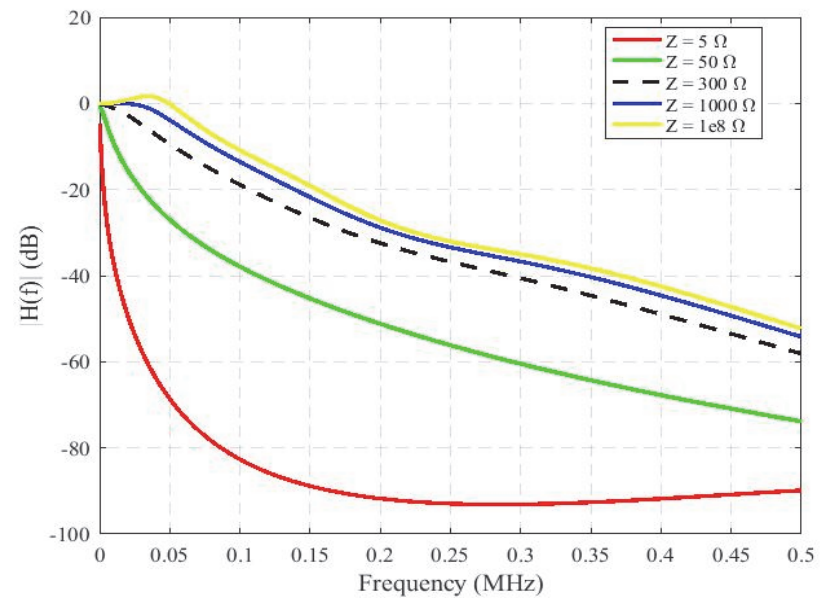

Figure 3 Influence of the terminal load impedance on the CTF for the first scenario (magnitude response)

Fig. 3, Fig.4, Fig. 5 and Fig. 6 show the influence of the terminal load impedance on the CTF. When increasing the terminal load impedance, the attenuation decreases. When load is resistive or inductive, the CTF has a low pass characteristic with a continuous attenuation without selectivity in the narrowband [28].

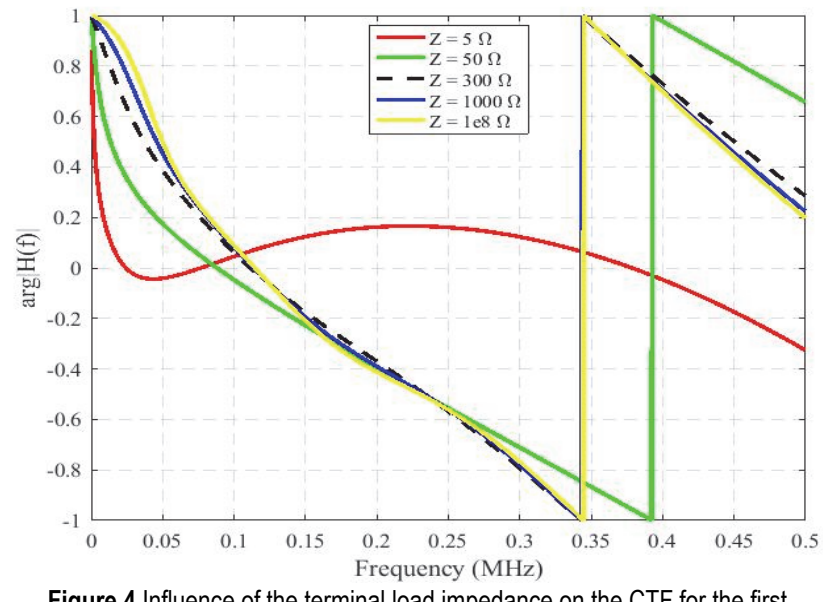

Figure 4 Influence of the terminal load impedance on the CTF for the first scenario (phase response)

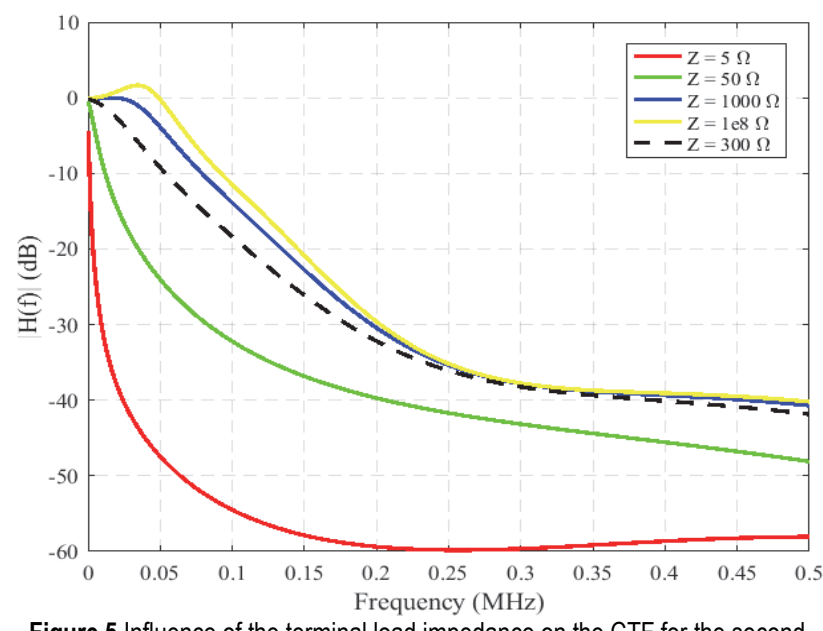

Figure 5 Influence of the terminal load impedance on the CTF for the second scenario (magnitude response)

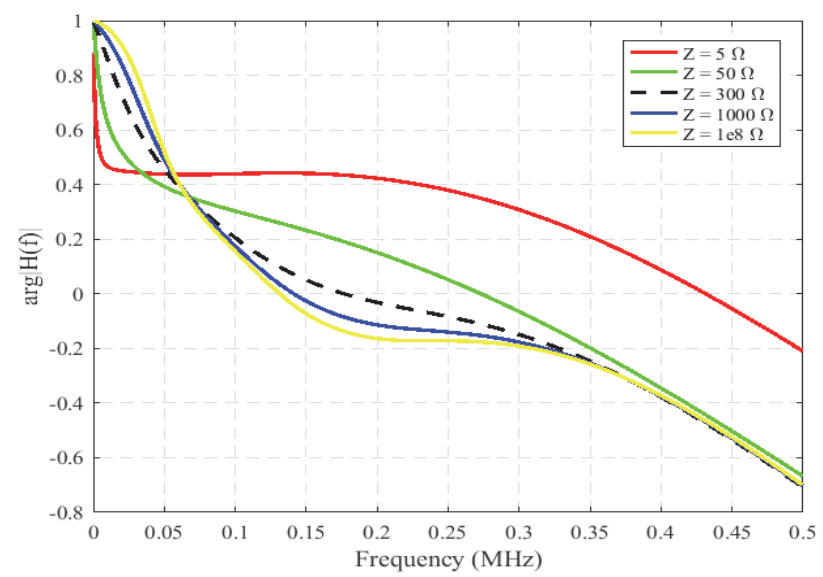

Figure 6 Influence of the terminal load impedance on the CTF for the second scenario (phase response)

\subsection{Influence of the Cross Section and Line Type}

In this scenario, the influence of the line type between the transmitting and the receiving node on the CTF and channel capacity was examined. For this case, the n68 node was selected as the receiving node. The cabling of the 
entire topology was intentionally changed to only one line type. The original topology with three types of cabling was also included in the comparison as well. Five types of cables were compared (underground NAYY $4 \times 150$, AYKY $3 \times 120+70$ and overhead AlFe $4 \times 50, \mathrm{X} 00 / 0-\mathrm{A}$ $4 \times 16, \mathrm{X} 00 / 0-\mathrm{A} 3 \times 70+71,5)$. The four-sector cable NAYY is already implemented in NS-3 PLC simulator. Other types of cables were modelled in the same manner in the NS-3 PLC simulator using necessary data provided in $[31,32]$. Fig. 7 and Fig. 8 show the influence of the cross section and line type on the CTF.

The highest attenuation can be observed for overhead line AlFe $4 \times 50$. This is the oldest type of overhead line and these results were expected because the insulation for these lines is air only. They suffer from many problems such as extreme weather conditions, brownout, load technical unbalance, phase load unbalance, low current earth leakages, over current and harmonics, high impedance faults in distribution networks [33].

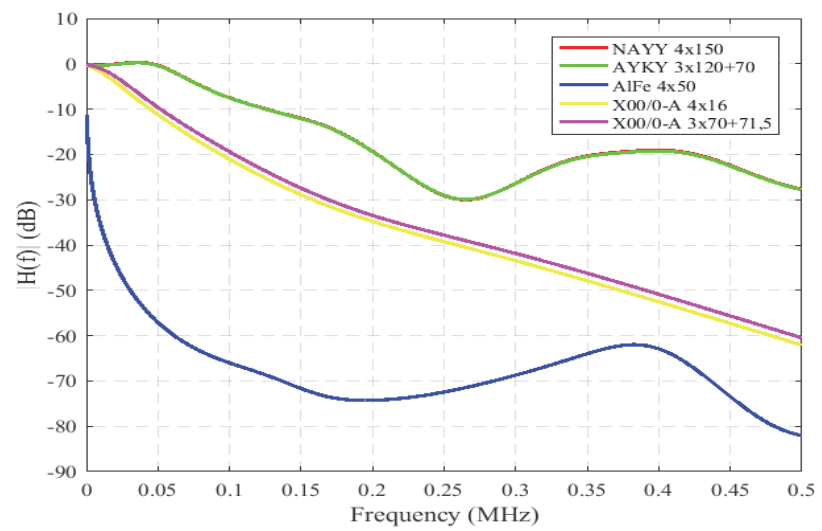

Figure 7 Influence of the line type on the CTF (magnitude response)

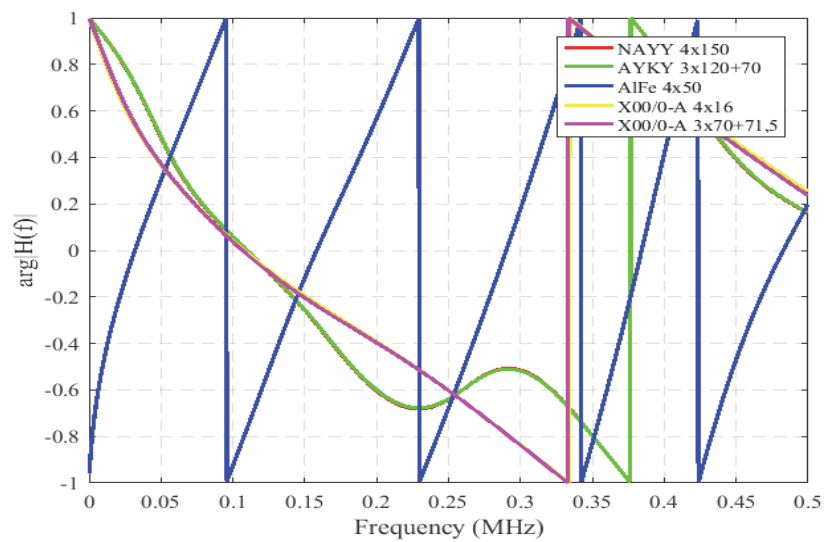

Figure 8 Influence of the line type on the CTF (phase response)

The modern solution of the AlFe overhead line is X00/0-A type of line with the polyethylene as the insulation. Underground cables NAYY and AYKY have similar characteristics and are at least attenuated lines. They have significant thermal capacity, which permits fewer overloading for long route applications [34]. According to [9], AMI applications over underground LV networks can cover distances up to $300 \mathrm{~m}$. The original topology, which contains overhead lines, has increased attenuation over the entire frequency range because it consists of different cross section of similar overhead lines.

Another purpose of this scenario was to show how the use of different types of lines has an impact on the channel link capacity. Tab. 1 shows the channel capacity for different line types in the CENELEC A and FCC band.

Table 1 Channel capacity for different line types in the CENELEC A and FCC band

\begin{tabular}{|c|c|c|}
\hline & \multicolumn{2}{|c|}{ Channel capacity on Physical Layer } \\
\hline Cable type & $\begin{array}{c}\text { CENELEC A band / } \\
\text { kbps }\end{array}$ & FCC band / Mbps \\
\hline NAYY 4 150 & 111,87 & 0,3206 \\
\hline AYKY 3 $120+70$ & 110,87 & 0,3205 \\
\hline AlFe 4 $\times 50$ & 51,82 & 0,3152 \\
\hline X00/0-A 4 16 & 56,44 & 0,3153 \\
\hline X00/0-A 3 $\times 70+71,5$ & 58,56 & 0,3154 \\
\hline real topology & 58,87 & 0,3154 \\
\hline
\end{tabular}

These results are in agreement with the results shown in Fig. 7 and Fig. 8 and are sufficient for the application which requires $2.4 \mathrm{kbps}$ in the G3-PLC communication [11].

\subsection{Influence of the Distance Between the Transmitter and the Receiver}

For this scenario, the distance between the transmitter and the receiver was varied by selecting additional three nodes from topology as receivers (n5, n14 and n45). The effect of changing the distance on the CTF was examined. Fig. 9 and Fig. 10 show a comparison of transmission functions for each pair of transmitter-receiver nodes. The original topology and line types were considered in this simulation. According to Fig. 9, it is obvious that node n68 has the highest attenuation because it is the most distant node from the transmitter node $\mathrm{n} 1$.

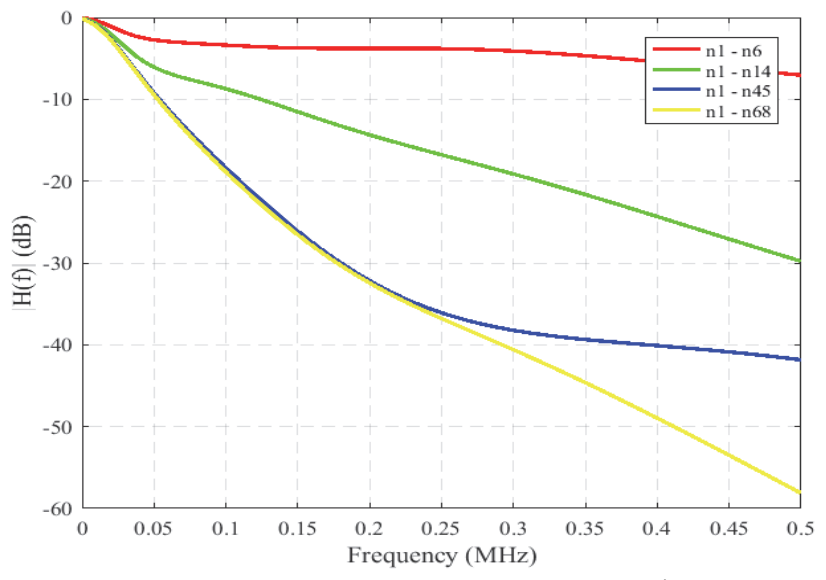

Figure 9 Distance between the transmitter and the receiver (magnitude response)

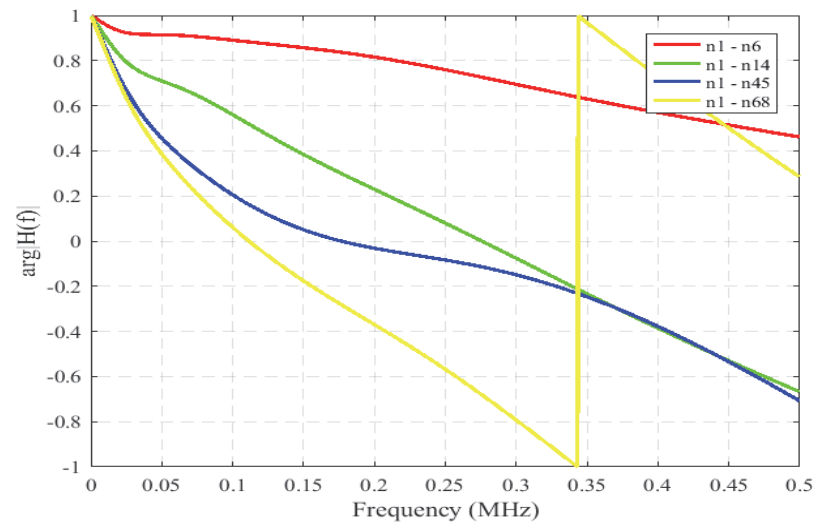

Figure 10 Distance between the transmitter and the receiver (phase response) 


\subsection{Influence of Background Noise and Modulation Type}

The aim of this scenario was to investigate the influence of different white noise values on the channel capacity. According to literature references [11, 23, 35], measured background noise varies and has different values for low and high, overhead and underground voltage lines. For the purpose of this simulation, three different experimental values of PSD of noise were chosen. The distance between the transmitter and the receiver was also changed to see the effect on the channel capacity. Tab. 2 shows the channel capacity for each pair of transmitterreceiver nodes and for different values of PSD noise.

Table 2 Channel capacity for different values of PSD noise

\begin{tabular}{|c|c|c|c|}
\hline \multirow{2}{*}{$\begin{array}{c}\text { Measuring } \\
\text { point } \\
\text { (distance) }\end{array}$} & $1 \mathrm{e}-9$ & $1 \mathrm{e}-10$ & $1 \mathrm{e}-12$ \\
\cline { 2 - 4 } & $825,35 \mathrm{kbps}$ & $2,84 \mathrm{Mbps}$ & $2,93 \mathrm{Mbps}$ \\
\hline $\begin{array}{c}\mathrm{n} 1 \rightarrow \mathrm{n} 5 \\
(59 \mathrm{~m})\end{array}$ & $588,88 \mathrm{kbps}$ & $1,05 \mathrm{Mbps}$ & $2,78 \mathrm{Mbps}$ \\
\hline $\begin{array}{c}\mathrm{n} 1 \rightarrow \mathrm{n} 14 \\
(168 \mathrm{~m})\end{array}$ & $524,46 \mathrm{kbps}$ & $742,23 \mathrm{kbps}$ & $1,40 \mathrm{Mbps}$ \\
\hline $\begin{array}{c}\mathrm{n} 1 \rightarrow \mathrm{n} 45 \\
(229 \mathrm{~m})\end{array}$ & $523,16 \mathrm{kbps}$ & $735,80 \mathrm{kbps}$ & $1,36 \mathrm{Mbps}$ \\
\hline $\begin{array}{c}\mathrm{n} 1 \rightarrow \mathrm{n} 68 \\
(278 \mathrm{~m})\end{array}$ & & & \\
\hline
\end{tabular}

According to the results presented in Tab. 2, the PSD noise value greatly affects the transmission capacity. As the value of PSD decreases, the channel capacity increases resulting in better transmission characteristics. It is also interesting to see what impact on transmission capacity have different modulations. The result of simulations in NS-3 PLC simulator is shown in Tab. 3.

Table 3 Channel capacity for different modulation type

\begin{tabular}{|c|c|c|c|c|}
\hline Modulation & 32-PSK & 4-QAM & 16-QAM & 64-QAM \\
\hline Capacity / kbps & 523,16 & 364,41 & 533,76 & 535,94 \\
\hline
\end{tabular}

As can be seen, higher transmission capacities can be achieved with QAM modulation. But these are only theoretical values regardless of BER. Certain types of modulations are more susceptible to interference in the real environment and need a greater SNR [34].

\section{MEASUREMENTS IN REAL POWER NETWORK}

All measurements were performed using Iskraemeco SPY maintenance tool for second generation of PLC networks. It provides the information about signal to noise level on first and second carrier - channel, power level of received signal, electrical phase position of received communication signal, FER and average of the last 25 measurements of noise floor in the CENELEC A band.

The measurements were conducted between node $\mathrm{n} 1$ and two measurement points, n45 and n68. These points present smart meters at customer point of supply. Tab. 4 shows average values of noise floor, SNR on first and second carrier, FER, power level on first and second carrier and electrical phase position. Some values were not provided because the device could not measure them so these are locations with unsuccessful measurement readings.

\begin{tabular}{|c|c|c|c|c|c|c|c|c|}
\hline 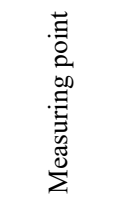 & 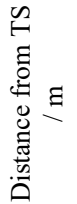 & 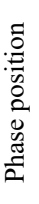 & 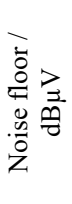 & $\frac{\partial^{\circ}}{\frac{\alpha}{11}}$ & 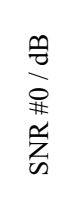 & 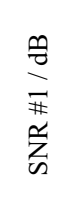 & 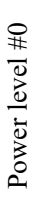 & $\begin{array}{l}\text { \# } \\
0 \\
\text { d } \\
\overline{0} \\
0 \\
0\end{array}$ \\
\hline $\begin{array}{c}\text { Smart } \\
\text { meter n68 }\end{array}$ & 278 & 0 & 66 & - & 21,45 & 17,54 & - & - \\
\hline $\begin{array}{c}\text { Smart } \\
\text { meter n } 45\end{array}$ & 229 & 0 & 66 & 18 & 32,03 & 30,12 & - & - \\
\hline
\end{tabular}

By analyzing the situational plan, the distances of measurement points from TS, and the results of simulations for SNR (see Fig. 11), it can be concluded that the distances are within the values defined by standards and technical specifications of the meters. The noise floor levels are also within the permissible limits. The reason why this communication was unsuccessful was occasional impulse interference that could not be located due to its occasional occurrence. The occurrence of this type of interference indicates the occasional use of certain home appliances. A possible reason for this situation is the fact that there is no obligation to comply with the standard for consumers.

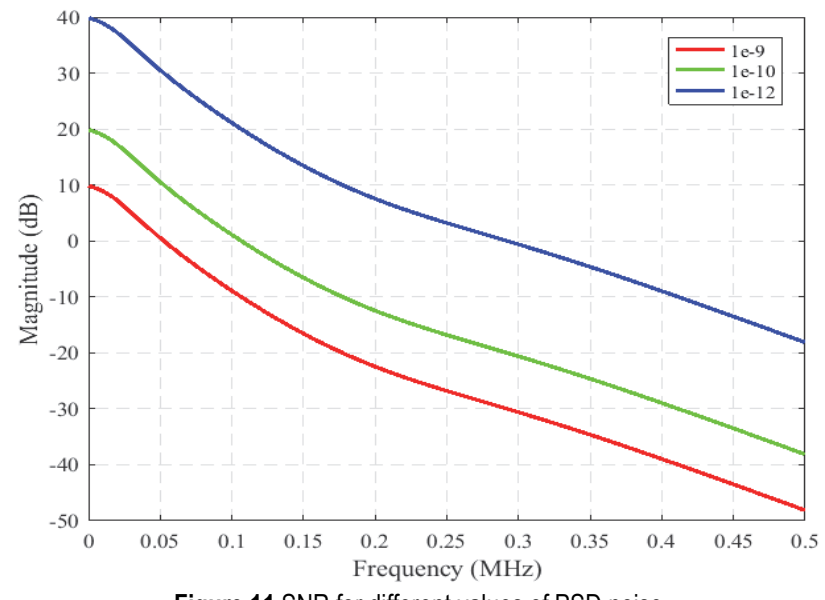

Figure 11 SNR for different values of PSD noise

\section{DISCUSSION}

Simulations were carried out to show the effect of different parameters of power line topology on PLC system. The overhead LV distribution network was modelled in the extended frequency range. According to the available literature, all other simulation parameters have been adjusted. The influence of the terminal load impedance was investigated. It was concluded that with terminal impedance larger than characteristic impedance of power line, the attenuation decreases. According to [16], it is important to emphasize that the attenuation of notches decreases for cases of terminal impedance having values less than or greater than characteristic impedance of power line. Different cross section and line type between the transmitting and the receiving node on the CTF and channel capacity were also examined. As expected, the highest attenuation is for overhead line AlFe. These results were expected because the insulation for these lines is air only. The original topology has increased attenuation over the entire frequency range because it consists of different cross section of similar overhead lines. Underground 
cables have similar characteristics and are at least attenuated lines. These results are in agreement with the results for channel capacity on physical layer. It shows that channel capacity for underground lines is higher than overhead lines and is similar for different types of underground/overhead lines. As it was concluded before, these cables suffer from many problems which cause lower values of channel capacity. The distance between the transmitter and the receiver was varied by selecting additional nodes from topology as receivers. The effect of changing the distance on the CTF showed that the most distant node has the highest attenuation. For the purpose of investigation how different background noise and modulation type influence channel capacity, three different experimental values of PSD of noise were chosen. According to the results as value of PSD decreases the channel capacity increases resulting in better transmission characteristic. It was concluded that higher transmission capacities can be achieved with QAM modulation.

The measurements were conducted between node $\mathrm{n} 1$ and two measurement points, n45 and n68. Although the distances from TS, noise floor levels and the results of simulations are within the values defined by standards and technical specifications of the meters, these are locations with unsuccessful measurement readings. The reason for this was occasional impulse interference that could not be located which implies the occasional use of certain home appliances.

\section{CONCLUSION}

In this article, the influence of the parameters such as terminal load impedances, direct line length from transmitter to receiver, cable type, background noise and modulation type on the CTF and channel link capacity were analysed over the frequency range $0-500 \mathrm{kHz}$. Although the line type used in this article presents a modern solution of the AlFe overhead line, the original topology has increased attenuation over the observed frequency range. Previous article results show that underground cables are at least attenuated lines for longer distances. Repeaters are also used to overcome problem with signal strength. There are several things that need to be addressed which would help to get more realistic results from simulations. The value of the terminal load impedance depends on the connected electrical devices as well as on the frequency in the power network. In order to get more accurate results, the values of the impedances should be selected according to a real environment. Noise is another factor that affects the simulation results as well as the quantity of transmitted data. Impulse noise component has large impact on PLC network and it is important to minimize its effect. It was concluded that beside the impact of topological parameters and physical characteristics of the power line on the performance of PLC system, there are different interference factors which affect the reliability of communication. Field measurements showed that although parameters are within the values defined by standards and technical specifications there are points in the power network with unsuccessful measurement readings. Therefore it is very important to have well defined standards for connected devices to the power network and to implement them strictly. It is a big challenge that should be overcome especially in countries where standards are not consistently applied.

\section{List of abbreviation}

PLC - Power Line Communication

LV - Low Voltage

EMI - Electro Magnetic Interference

NIE - Non-Intentional Emissions

DG - Distributed Generation

EV - Electric Vehicles

UNB - Ultra-Narrow Band

NB - Narrow Band

LDR - Low Data Rate

HDR - High Data Rate

BB - Broadband

FCC - Federal Communications Commission

ARIB - Association of Radio Industries and Businesse

IoT - Internet of Things

PRIME - Powerline Intelligent Metering Evolution

BER - Bit Error Rate

FSK -Frequency Shift Keying

SFSK -Spread Frequency Shift Keying

OFDM - Orthogonal Frequency Division Multiplexing

TLT - Transmission Line Theory

NS-3 -Network Simulator 3

SM - Smart Metering

MV - Medium Voltage

HV - High Voltage

CTF - Channel Transfer Function

SNR - Signal-to-Noise Ratio

PSD - Power Spectral Density

TS - Transformer Substation

AMI - Advanced Metering Infrastructure

QAM - Quadrature Amplitude Modulation

FER -Frame Error Rate

\section{REFERENCES}

[1] Nassar, M., Lin, J., Mortazavi, Y., Dabak, A., Kim, I., \& Evans, B. (2012). Local Utility Power Line Communications in the 3 - $500 \mathrm{kHz}$ Band: Channel Impairments, Noise, and Standards. IEEE Signal Processing Magazine, 29(5), 116127. https://doi.org/10.1109/msp.2012.2187038

[2] Lopez, G., Matanza, J., Vega, D. D. L., Castro, M., Arrinda, A., Moreno, J. I., \& Sendin, A. (2019). The Role of Power Line Communications in the Smart Grid Revisited: Applications, Challenges, and Research Initiatives. IEEE Access, 7, 117346-117368. https://doi.org/10.1109/access.2019.2928391

[3] Cano, C., Pittolo, A., Malone, D., Lampe, L., Tonello, A. M., \& Dabak, A. G. (2016). State of the Art in Power Line Communications: From the Applications to the Medium. IEEE Journal on Selected Areas in Communications, 34(7), 1935-1952. https://doi.org/10.1109/jsac.2016.2566018

[4] Kabalci, E. \& Kabalci, Y. (2019). Smart Grids and Their Communication Systems. Singapore: Springer. https://doi.org/10.1007/978-981-13-1768-2

[5] Ferreira, H. C. (2010). Power line communications: theory and applications for narrowband and broadband communications over power lines. Chichester, West Sussex, UK: Wiley. https://doi.org/10.1002/9780470661291 
[6] Ikpehai, A., Adebisi, B., \& Rabie, K. (2016). Broadband PLC for Clustered Advanced Metering Infrastructure (AMI) Architecture. Energies, 9(7), 569. https://doi.org/10.3390/en9070569

[7] Liu, W., Sigle, M., \& Dostert, K. (2011). Channel characterization and system verification for narrowband power line communication in smart grid applications. IEEE Communications Magazine, 49(12), 28-35. https://doi.org/10.1109/MCOM.2011.6094003

[8] Sharma, K. \& Saini, L. M. (2017). Power-line communications for smart grid: Progress, challenges, opportunities and status. Renewable and Sustainable Energy Reviews, 67, 704-751. https://doi.org/10.1016/j.rser.2016.09.019

[9] Kim, I. H., Kim, W., Park, B., \& Yoo, H. (2014). Channel measurements and field tests of narrowband power line communication over Korean underground LV power lines. 18th IEEE International Symposium on Power Line Communications and Its Applications. https://doi.org/10.1109/isplc.2014.6812361

[10] Arechalde, I., Castro, M., Garcia-Borreguero, I., Sendin, A., Urrutia, I., \& Fernandez, A. (2017). Performance of PLC communications in frequency bands from $150 \mathrm{kHz}$ to 500 kHz. 2017 IEEE International Symposium on Power Line Communications and Its Applications (ISPLC). https://doi.org/10.1109/isplc.2017.7897123

[11] Elfeki, I., Jacques, S., Aouichak, I., Doligez, T., Raingeaud, Y., \& Bunetel, J.-C. L. (2018). Characterization of Narrowband Noise and Channel Capacity for Powerline Communication in France. Energies, 11(11), 3022. https://doi.org/10.3390/en11113022

[12] Borovina, D., Mujcic, A., Zajc, M., \& Suljanovic, N. (2018) Investigation of Narrow-Band Power-Line Carrier Communication System Performance in Rural Distribution Grids. Elektronika Ir Elektrotechnika, 24(1). https://doi.org/10.5755/j01.eie.24.1.20149

[13] Mlynek, P., Misurec, J., Silhavy, P., Fujdiak, R., Slacik, J., \& Hasirci, Z. (2019). Simulation of Achievable Data Rates of Broadband Power Line Communication for Smart Metering. Applied Sciences, 9(8), 1527. https://doi.org10.3390/app9081527

[14] Najjar, S., Rouissi, F., Vinck, A. J. H., Gassara, H., \& Ghazel, A. (2018). SFSK versus OFDM Performances under the Narrowband PLC Channel Impairments. 2018 6th International Conference on Multimedia Computing and Systems (ICMCS). https://doi.org/10.1109/icmcs.2018.8525990

[15] Masood, B., Nazar, W., \& Masood, R. (2018). Channel Modeling of Low Voltage NB-PLC Network using Statistical and Deterministic Channel Modeling Approaches. 2018 7th International Conference on Renewable Energy Research and Applications (ICRERA). https://doi.org/10.1109/icrera.2018.8566732

[16] Mlynek, P., Hasirci, Z., Misurec, J., \& Fujdiak, R. (2016). Analysis of Channel Transfer Functions in Power Line Communication System for Smart Metering and Home Area Network. Advances in Electrical and Computer Engineering, 16(4), 51-56. https://doi.org/10.4316/aece.2016.04008

[17] Marrocco, G., Statovci, D., \& Trautmann, S. (2013). A PLC broadband channel simulator for indoor communications. 2013 IEEE 17th International Symposium on Power Line Communications and Its Applications. https://doi.org/10.1109/isplc.2013.6525871

[18] Canete, F., Cortes, J., Diez, L., \& Entrambasaguas, J. (2011). A channel model proposal for indoor power line communications. IEEE Communications Magazine, 49(12), 166-174. https://doi.org/10.1109/mcom.2011.6094022

[19] Khyari, N., Khalfallah, S., Barouni, Y., \& Slama, J. B. H. (2018). Comparative Performance Analysis of Cooperative
Outdoor PLC. 2018 IEEE/ACS 15th International Conference on Computer Systems and Applications (AICCSA). https://doi.org/10.1109/aiccsa.2018.8612794

[20] Aalamifar, F., Schlogl, A., Harris, D., \& Lampe, L. (2013). Modelling power line communication using network simulator-3. 2013 IEEE Global Communications Conference (GLOBECOM) https://doi.org/10.1109/glocom.2013.6831526

[21] Lampe, L., Tonello, A. M., \& Swart, T. G. (2016). Power line communications principles, standards and applications from multimedia to smart grid. Chichester (Inglaterra): John Wilder \& Sons. https://doi.org/10.1002/9781118676684

[22] Ndjiongue, A., \& Ferreira, H. (2019). Power line communications (PLC) technology: More than 20 years of intense research. Transactions on Emerging Telecommunications Technologies, 30(7). https://doi.org/10.1002/ett.3575

[23] Le, J., Wang, C., Zhou, W., Liu, Y. Y., \& Cai, W. (2017). A novel PLC channel modeling method and channel characteristic analysis of a smart distribution grid. Protection and Control of Modern Power Systems, 2(1). https://doi.org/10.1186/s41601-017-0044-2

[24] Misurec, J., Mlynek, P., \& Bezzateev, S. (2017). The modeling of power line for PLC in smart grids. 2017 Progress In Electromagnetics Research Symposium - Spring (PIERS). https://doi.org/10.1109/piers.2017.8261848

[25] Mlynek, P., Misurec, J., Koutny, M., Fujdiak, R., \& Jedlicka, T. (2015). Analysis and Experimental Evaluation of Power Line Transmission Parameters for Power Line Communication. Measurement Science Review, 15(2), 6471. https://doi.org/10.1515/msr-2015-0010

[26] Gotz, M., Rapp, M., \& Dostert, K. (2004). Power line channel characteristics and their effect on communication system design. IEEE Communications Magazine, 42(4), 7886. https://doi.org/10.1109/mcom.2004.1284933

[27] Khach, E., Jacobsen, K. E., Skov, M. N., Højholt, N. B., \& Sørensen, R. B. (2014). Investigation of QoS in Plc and evaluation of a Ns-3 based Plc simulator. Retrieved from http://kom.aau.dk/group/14gr631/Report.pdf

[28] Alaya, R. \& Attia, R. (2017). Characterization of low voltage access network for narrowband powerline communications. 2017 25th International Conference on Software, Telecommunications and Computer Networks (SoftCOM). https://doi.org/10.23919/softcom.2017.8115514

[29] Canete, F., Cortes, J., Diez, L., \& Entrambasaguas, J. (2011). A channel model proposal for indoor power line communications. IEEE Communications Magazine, 49(12), 166-174. https://doi.org/10.1109/mcom.2011.6094022

[30] González-Sotres, L., Frías, P., \& Mateo, C. (2017). Power line communication transfer function computation in realnetwork configurations for performance analysis applications. IET Communications. https://doi.org/10.1049/iet-com.2016.0135

[31] Nagendra, A. \& Saini, L. M. (2018). A Review on Powerline Carrier Channels for both Overhead Lines and Underground Cables. 2018 IEEE International Students Conference on Electrical, Electronics and Computer Science (SCEECS). https://doi.org/10.1109/sceecs.2018.8546994

[32] Papadopoulos, T. A., Kaloudas, C. G., Chrysochos, A. I., \& Papagiannis, G. K. (2013). Application of Narrowband Power-Line Communication in Medium-Voltage Smart Distribution Grids. IEEE Transactions on Power Delivery, 28(2), 981-988. https://doi.org/10.1109/tpwrd.2012.2230344

[33] Suljanović, N., Mujčić, A., Zajc, M., \& Tasič, J. F. (2004). Approximate computation of high-frequency characteristics for power line with horizontal disposition and middle-phase to ground coupling. Electric Power Systems Research, 69(1), 17-24.https://doi.org/10.1016/j.epsr.2003.07.005 
[34] Peretto, L., Tinarelli, R., Bauer, A., \& Pugliese, S. (2011). Fault location in underground power networks: A case study. Isgt 2011. https://doi.org/10.1109/isgt.2011.5759198

[35] Mlynek, P., Misurec, J., Toman, P., Silhavy, P., Fujdiak, R., Slacik, J., Hasirci, Z., \& Samouylov, K. (2018). Performance Testing and Methodology for Evaluation of Power Line Communication. Elektronika Ir Elektrotechnika, 24(3). https://doi.org/10.5755/j01.eie.24.3.20983

\section{Contact information:}

Dženana TOMAŠEVIĆ, PhD student

(Corresponding author)

University of Sarajevo,

Faculty of Electrical Engineering,

Zmaja od Bosne bb, 71000 Sarajevo

E-mail: dzenana.tomasevic@etf.unsa.ba

Tatjana KONJIĆ, Professor

University of Tuzla,

Faculty of Electrical Engineering

Franjevačka 2, 75000 Tuzla

E-mail: tatjana.konjic@untz.ba

Suad KASAPOVIĆ, Professo

University of Tuzla,

Faculty of Electrical Engineering

Franjevačka 2, 75000 Tuzla

E-mail: suad.kasapovic@untz.ba 